\title{
Symbol-Rate Dependence of Dominant Nonlinearity and Reach in Coherent WDM Links
}

\author{
Nicola Rossi, Paolo Serena, Member, IEEE, and Alberto Bononi, Senior Member, IEEE
}

\begin{abstract}
We provide simulations of the nonlinear threshold (NLT) versus per-channel symbol rate at a constant bandwidth efficiency for a 15-channel wavelength-division multiplexing long-haul coherent optical transmission over a $20 \times 100 \mathrm{~km}$ singlemode fiber link, both dispersion-managed (DM) and dispersionuncompensated (DU), with several popular polarization multiplexed modulation formats. We exploit the nonlinearity-decoupling method to estimate the NLT due to each individual nonlinear effect, and, thus, show how the dominant nonlinearity changes with symbol rate. Plots of the NLT normalized to the symbol rate also reveal the symbol rate achieving the longest distance. Assuming a signal-independent Gaussian nonlinear interference, we derive a new formula yielding the system reach based on knowledge of NLT measurements on a much shorter link. We finally provide reach simulations to verify the accuracy of the new reach formula for both DM and DU links.
\end{abstract}

Index Terms-Coherent systems, nonlinear effects, PDM-BPSK, PDM-QPSK, PDM-16QAM.

\section{INTRODUCTION}

$\mathbf{T}$ $\mathrm{HE}$ issue of the best granularity in wavelength division multiplexed (WDM) long-haul coherent optical links, i.e., the per-carrier symbol rate for orthogonal frequency division multiplexing, and the per-channel symbol rate for single-carrier modulations, was recently discussed [1]-[9]. The best symbol rate either minimizes the bit error rate (BER) at a fixed distance, or maximizes the largest transparent transmission distance (i.e., the reach) at a fixed BER.

We previously reported on lengthy Monte-Carlo simulations of the nonlinear threshold (NLT) versus symbol rate $R$ in polarization-division-multiplexed (PDM) quadrature phase shift keying (QPSK) coherent systems at a constant bandwidth efficiency $\eta \triangleq R / \Delta f$, where $\Delta f$ is the channel spacing, with the aim of highlighting the dominant nonlinearity as the granularity changes [10]. In both [10] and in this paper, the NLT $P_{\mathrm{NLT}}$ is defined as the transmitted per-channel power at $1 \mathrm{~dB}$ signal to noise ratio (SNR) penalty at $\mathrm{BER}=10^{-3}$ (the integrated NLT $P_{\mathrm{NLT}} \cdot N$ is also often used, with $N$ the number of spans, see e.g., [11], [12]). We also reported on how the reach in dispersion-uncompensated (DU) links can be inferred from both the nonlinear interference (NLI) accumulation parameter $\epsilon$ and the power margin measured at a given transmission distance [13, eq. (24)]. The power margin is proportional to the ratio $P_{\mathrm{NLT}} / P_{\mathrm{L}}$, where the linear threshold $P_{\mathrm{L}}$ is the power at

Manuscript received September 8, 2014; revised January 9, 2015 and April 20, 2015; accepted April 21, 2015. Date of publication April 27, 2015; date of current version June 3, 2015.

The authors are with the Department of Information Engineering, Università degli Studi di Parma, Parma 43125, Italy (e-mail: rossi_n@tlc.unipr.it; paolo.serena@unipr.it; alberto.bononi@unipr.it).

Color versions of one or more of the figures in this paper are available online at http://ieeexplore.ieee.org.

Digital Object Identifier 10.1109/JLT.2015.2426680 the required SNR in linear propagation. Similar inferences for dispersion managed (DM) systems are well known (see, e.g., [14]). Since $P_{\mathrm{L}}$ scales linearly with $R$, the largest power margin (hence, as we shall see, the longest reach) is achieved at the symbol rate that maximizes the normalized margin $P_{\mathrm{NLT}} / R$.

In this paper, which is an extended version of [15], we report on simulations of $P_{\mathrm{NLT}} / R$ for both DM and DU systems, for several PDM single-carrier modulation formats of interest with non-return to zero (NRZ) supporting pulses, namely: binary phase-shift keying (PDM-BPSK), PDM-QPSK, and 16level quadrature amplitude modulation (PDM-16QAM). Compared to [15], we here extend the investigation to higher spectral efficiency, and to polarization switched QPSK (PS-QPSK) and interleaved return to zero (iRZ) for both PDM-BPSK and PDM-QPSK [16], [17]. All NLT simulations were performed for a $20 \times 100 \mathrm{~km}$ single-mode fiber (SMF) link with end-span lumped amplification and 15 WDM channels. The split-step Fourier method (SSFM) simulations were based on the Manakov nonlinear equation and used the nonlinearity-decoupling method proposed in [18] (which is reviewed for convenience in Appendix B) to yield the NLT due to each Kerr nonlinearity. We thus aim to shed light on the way the nonlinear Kerr effect manifests on several modulation formats propagating in both legacy DM and modern DU links. We present the NLT results in terms of the normalized margin versus $R$ to highlight both i) the dominant nonlinearity for each format, and ii) the optimal granularity for maximum reach.

For DU systems we double-check the margin dependence on symbol-rate against predictions of the Gaussian noise (GN) model [19], and observe a format-dependent offset, in agreement with [20]-[23]. Another novelty compared to [15] is that, by working with the GN theory, we come up with a new formula that relates the system reach to the NLI accumulation parameter $\epsilon$ and to the fictitious noise figure found in fixed-distance simulations at NLT. Surprisingly, we show that such a formula also very well predicts the reach of DM systems.

The paper is organized as follows. Section II briefly recalls the system design problem. Section III describes the simulations setup, while Section IV presents and discusses the simulations results. Section IV-C presents GN-model extrapolations of the margin/reach dependence on symbol-rate to a large channel count. Section $V$ presents the novel formula that quantifies the maximum reach as a function of the simulated fictitious noise figure at NLT, and checks it against simulations. Finally, Section VI contains the conclusions.

\section{SyStem DesigN}

The design of long-haul optical links is traditionally based on the maximization of the system reach, defined as the maximum distance bridged by a channel without regeneration. 


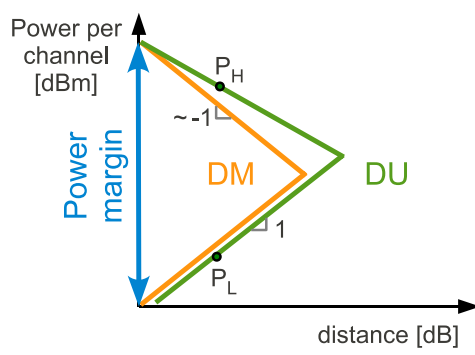

Fig. 1. Simplified sketch of received BER contours, plotted against power per channel and distance. $P_{\mathrm{H}}$ is the nonlinear line. $P_{\mathrm{L}}$ is the linear line. Their ratio is the power margin.

To this goal, it is customary to evaluate the reach from BER, or equivalently Q-factor, contours versus both launched power and distance (see, e.g., [13], [14], [24] and Fig. 8). A simplified straight-line sketch of such contours is given in Fig. 1 for both a DM and a DU system [25, presentation slides]. At any given reachable distance there are two powers yielding the target BER, the largest one $P_{\mathrm{H}}$ in the so called "nonlinear region," the other $P_{\mathrm{L}}$ in the "linear region." Their difference is the power margin. While the linear $P_{\mathrm{L}}$ line versus distance (or span number $N$ ) has slope $1 \mathrm{~dB} / \mathrm{dB}$ when the spans are identical, the negative slope of the nonlinear $P_{\mathrm{H}}$ line depends on the dispersion map. For DU links, theory shows the nonlinear line slope is $-(1+\epsilon) / 2$ $\mathrm{dB} / \mathrm{dB}$, where the NLI accumulation parameter $\epsilon$ is a small positive number $\ll 1$ [13], while for DM links the nonlinear slope is close to $-1 \mathrm{~dB} / \mathrm{dB}$ [14], or equivalently $\epsilon \cong 1$. The reason of the larger negative nonlinear slope in DM links can be traced back to the nonlinear contributions of the various spans: in DM systems they are unequal and strongly correlated, while in DU systems they tend to be more even and uncorrelated by the large cumulated dispersion [26]. The linear line $P_{\mathrm{L}}$ of the DU link lays below the one of the DM link because of the absence of lossy in-line dispersion compensating fibers in the DU span. Together with the smaller tilt of the nonlinear line $P_{\mathrm{H}}$, this gives the DU link a longer reach than the DM link.

The question is now how these BER contours and thus the reach change as the symbol rate $R$ is changed. It is clear from Fig. 1 that, assuming $\epsilon$ independent of $R$, by maximizing the power margin $P_{\mathrm{H}} / P_{\mathrm{L}}$ one also maximizes the reach. Appendix A provides an analytical proof of the above fact for DU links. Since $P_{\mathrm{L}}$ is proportional to the receiver bandwidth, and hence to $R$, then the ratio $P_{\mathrm{H}} / R$ should be maximized. In DU links, $P_{\mathrm{NLT}}$ versus $N$ (in a dB-dB plot) is known to be approximately a straight-line, placed $3.42 \mathrm{~dB}$ below $P_{\mathrm{H}}[13$, Fig. 8]. Hence in the next section we provide a thorough investigation by simulation of the normalized margin $P_{\mathrm{NLT}} / R$ at a $\mathrm{BER}=10^{-3}$ in both DM and DU links and for several popular modulation formats.

\section{Simulated SYSTEMS}

Fig. 2 shows the optical systems simulated with the opensource software Optilux [27]. The transmitter consisted of $N_{\text {ch }}=15 \mathrm{WDM}$ channels modulated at a symbol rate of $R$ Gbaud with channel spacing $\Delta f$ and bandwidth efficiency $R / \Delta f=0.56$ (except where otherwise stated). We considered four modulation formats: PDM-BPSK, PDM-QPSK, PS-QPSK,
$D M$

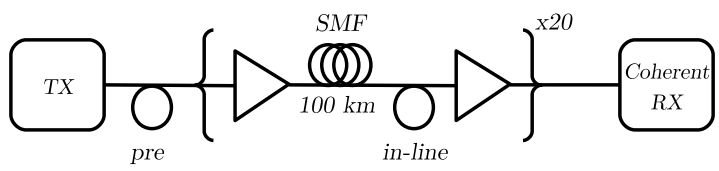

DU

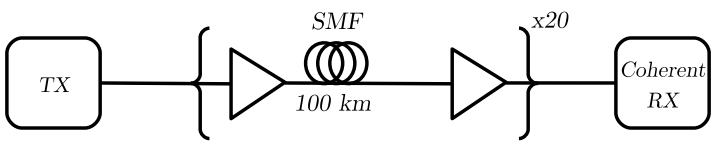

Fig. 2. Block diagrams of DM and DU simulated links. TX block represents 15 WDM channels with spacing $\Delta f=R / \eta$, with $R$ the symbol rate and $\eta$ the bandwidth efficiency. In DM we use a RDPS of $30 \mathrm{ps} / \mathrm{nm}$ and a pre-compensation of $-540 \mathrm{ps} / \mathrm{nm}$.

and PDM-16QAM with differential phase encoding/decoding. For all formats we used NRZ supporting pulses, and for PDMBPSK and PDM-QPSK we also considered iRZ pulses with $50 \%$ duty cycle [16], [17]. Before multiplexing, each channel was modulated with independent random symbols and filtered by a second order super-Gaussian filter of bandwidth $\Delta f$. The number of symbols was a function of $R$, and sized to correctly reproduce the maximum walk-off cumulated by the edge channels of the WDM comb with respect to (w.r.t.) the central channel. In all cases it was confined between 1024 and 16384 symbols. The transmission line consisted of 20 spans of $100 \mathrm{~km}$ of SMF (dispersion $D=17 \mathrm{ps} / \mathrm{nm} / \mathrm{km}$, slope parameter $\beta_{3}=0$, attenuation $\alpha=0.2 \mathrm{~dB} / \mathrm{km}$, nonlinear coefficient $\gamma=1.3 \mathrm{~W}^{-1} \cdot \mathrm{km}^{-1}$ ). At the end of the link, lumped electronic dispersion compensation was implemented in the coherent receiver. In the DM case, we used an in-line residual dispersion per span (RDPS) of $30 \mathrm{ps} / \mathrm{nm}$ and a straight-line rule pre-compensation of $-540 \mathrm{ps} / \mathrm{nm}$ [28], while in the DU case no compensation fiber was used. Propagation used the vector SSFM with zero polarization mode dispersion and Manakov nonlinear step [29], [30]. The maximum nonlinear phase per step was $3 \cdot 10^{-3}$ rad at symbol rates lower than 80 Gbaud, and $6 \cdot 10^{-4}$ rad at higher symbol rates. Before detection we used a sixth order Butterworth optical filter of bandwidth $\Delta f$ to extract the central WDM channel. The signal was then converted to an electrical current by an ideal coherent mixer (zero laser phase noise, zero frequency offset) and sampled at two samples per symbol over a bandwidth of $0.6 R$. The discrete sequence was then digitally processed by performing polarization recovery and equalization with a 15-tap least-squares trained equalizer, and a 27-tap carrier phase estimation (CPE). For PSK modulation formats the CPE was performed with the Viterbi and Viterbi algorithm, while for 16QAM we used a blind-phase search algorithm [31].

The objective of the simulations was to estimate the $1-\mathrm{dB}$ constrained NLT $P_{\mathrm{NLT}}$ (see Appendix A) of the worst-case central channel versus symbol rate when nonlinear effects are selectively activated (see Appendix B). The procedure to estimate $P_{\text {NLT }}$ was the following [32, Algorithm 1]: first, we estimated the $\mathrm{SNR} S_{0}$ at the receiver sampler yielding $\mathrm{BER}=10^{-3}$ in linear transmission; second, we activated the Kerr nonlinearity of interest and varied the input power $P$ as well as the ASE power $N_{\mathrm{ASE}}=P /\left(1.26 \cdot S_{0}\right)$ (so as to enforce a linear SNR penalty of $\left.10 \log _{10}(1.26)=1 \mathrm{~dB}\right)$ until a $\mathrm{BER}=10^{-3}$ is again 

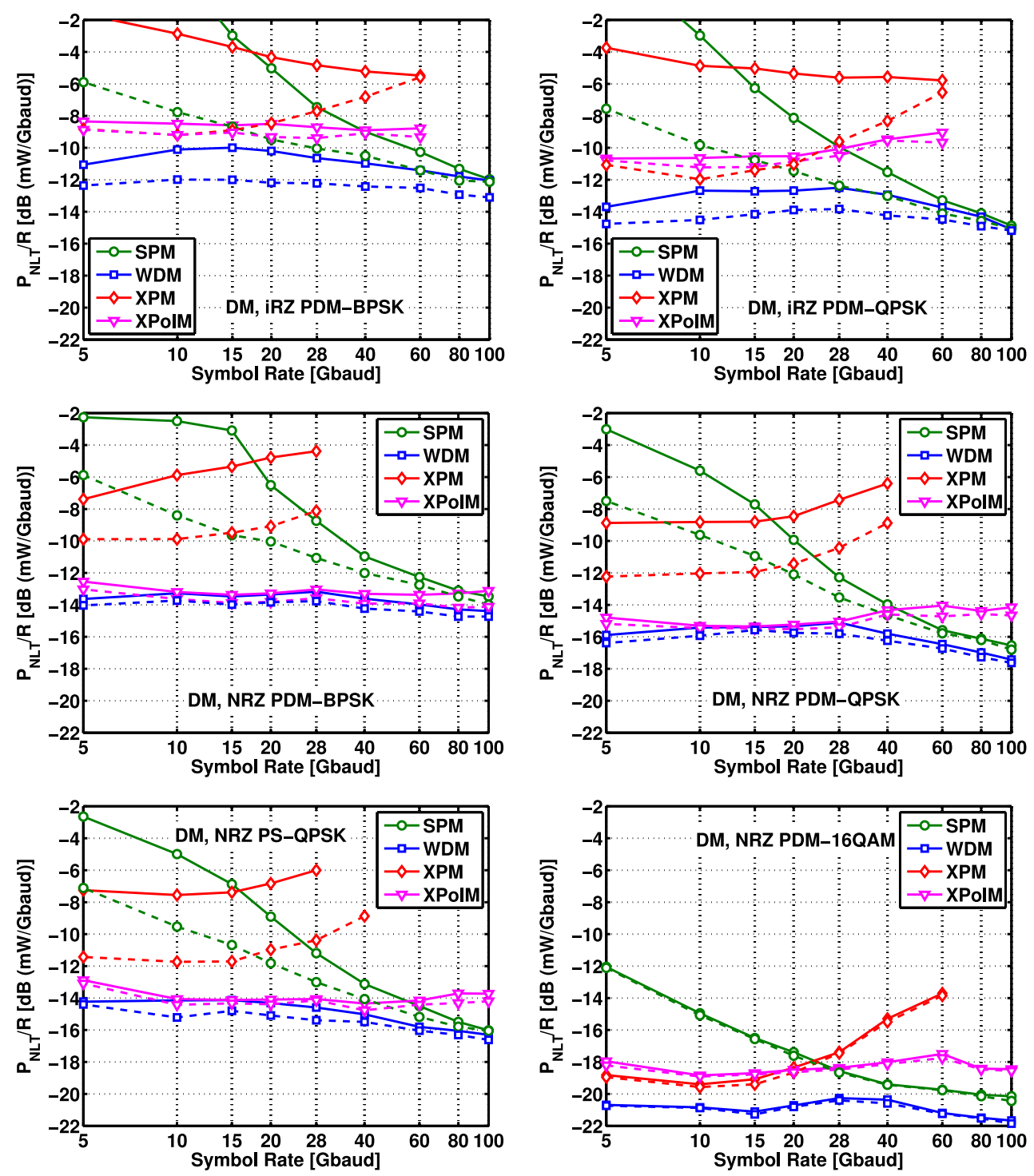

Fig. 3. Normalized margin $10 \log _{10}\left(P_{\mathrm{NLT}} / R\right)$ versus symbol rate $R$ [log scale] for a 15 -channel homogeneous WDM comb with $R / \Delta f=0.56$ over a $20 \times 100 \mathrm{~km}$ SMF DM link with RDPS $=30 \mathrm{ps} / \mathrm{nm}$. Solid lines: ASE loading at the receiver side. Dashed lines: distributed ASE. Labels: "SPM" = self-phase modulation only; "XPM" = scalar cross-phase modulation only; "XPolM" = cross-polarization modulation only; "WDM" = all nonlinearities.

obtained. The found value is $P_{\mathrm{NLT}}$. ASE power is changed by varying the lumped EDFA noise figure. ${ }^{1}$

Each BER estimation, with ASE either generated at each EDFA (distributed noise) or loaded at the end of the link (noise loading), was performed by Monte Carlo simulations, by repeating several independent transmissions of the WDM comb until 100 errors were counted. The input states of polarization (SOP) of the WDM carriers were randomly oriented over the Poincar sphere and randomly scrambled between two consecutive transmissions.

\section{NUMERICAL RESULTS}

Aim of this section is to quantify the NLT set by each Kerr nonlinearity for the most common coherent modulation formats. Recall that a small NLT corresponds to a strong nonlinearity,

\footnotetext{
${ }^{1}$ Other authors equivalently choose to vary the span loss, hence the EDFA gain [33].
}

and vice-versa. All results of simulations in the DM and DU systems described in Section III are summarized inFigs. 3-4, where the $1-\mathrm{dB}$ NLT $P_{\mathrm{NLT}}$ at $\mathrm{BER}=10^{-3}$, normalized to the symbol rate $R$, is plotted versus $R$. The reason for the normalization is that the maximum reach is a monotonic increasing function of the normalized margin $P_{\mathrm{NLT}} / R$, as discussed in the introduction and analytically proved for DU links in Appendix A. Hence the plots in Figs. 3 and 4 not only reveal the dominant nonlinear effect (yielding the lowest NLT), but also the symbol rate corresponding to the maximum reach.

In Figs. 3 and 4, in each labeled curve only the indicated nonlinearities are active, except for the curve labeled WDM, where all nonlinear effects are active and thus corresponds to the real case. The nonlinearities are: self-phase modulation (SPM), cross-phase modulation (XPM) and cross-polarization modulation (XPolM). The Appendix B summarizes the SSFM details for the selective activation of the desired nonlinearities. XPM and XPolM simulations were performed by the 
separate-field propagation (SFP) that misses classical four wave mixing (FWM), while WDM simulations used the unique-field propagation (UFP) which includes all Kerr nonlinearities. The XCI curve includes both XPM and XPolM and is added to better compare cross-channel to single-channel nonlinearity. In reading Figs. 3 and 4, please keep in mind that SPM and XPM are scalar effects that just depend on the field intensity.

\section{A. DM Link}

The six plots in Fig. 3 show the normalized margin versus symbol rate in the DM link. We report both the simplistic case of noise loading at the receiver (solid lines) and the true case of distributed ASE noise (dashed lines), where nonlinear signalASE interactions are fully accounted for. We will call noiseless $\mathrm{SPM} / \mathrm{XPM} / \mathrm{XPolM}$ those effects with ASE noise loading, and noisy SPM/XPM/XPolM those effects with distributed ASE. The six DM plots in Fig. 3 show that:

1) XPM with PSK formats is quite sensitive to signal-ASE interactions, since noisy and noiseless XPM-NLTs widely differ. This is due to the fact that in noiseless propagation in a DM link the symbol-rate quasi-periodic intensities of the transmitted PSK WDM fields are preserved, hence also the noiseless XPM field is symbol-rate quasi-periodic, and is thus well suppressed by the CPE block [34]. When in-line ASE is added, the intensity periodicity is broken and the XPM suppression in the CPE is way less effective, leading to a stronger residual XPM and thus a lower NLT.

Also, the low-pass filtering action introduced by fiber walk-off on the field intensity enhances XPM correlation on neighboring symbols, thus helping the phase tracking of the CPE [34]. In this case, shortening the CPE window may further improve the BER, however at the expense of an increase of the ASE-induced phase noise [35]. Since at the 1-dB NLT the ASE noise is largely dominant, ${ }^{2}$ then the number of CPE taps is generally set to a value large-enough ( 27 in our simulations) to well suppress ASE-induced phase fluctuations, and small-enough not to interfere with the frequency estimation block.

2) With homogeneous NRZ-PSK WDM formats, the dominant nonlinearity in the lower symbol rate range is XPolM. According to the XPolM model in [30], this is attributed to the fast modulation-induced random re-orientations of the mean WDM Stokes vector (called the pivot) in Stokes space, which cannot be tracked by the polarization demultiplexer at the receiver. ASE impact on XPolM is negligible, since ASE contribution to the pivot re-orientation is a second-order effect [10].

The XPolM-NLT is seen to be almost symbol-rate independent for all formats over the shown range. As symbol rate $R$ is increased at constant $\eta=R / \Delta f$, the channel spacing $\Delta f$ is also increased, and so is channel walk-off. XPM rapidly weakens and thus XPM-NLT increases ${ }^{3}$ with $R$ because of the low-pass intensity filtering due to walk-off [34]. XPolM is affected also by channels with a huge spectral distance from the channel of interest [38], [39] since it weakens with walk-off (i.e., with $R$ ) much more slowly than XPM. Hence the XPolM-NLT on the shown $R$ range appears almost flat.

\footnotetext{
${ }^{2}$ At the optimal power the ASE variance is twice that of the NLI [36], [37]. The 1-dB NLT is about 1dB below the optimal power [13].

${ }^{3}$ For the anomalous behavior of noiseless XPM-NLT for iRZ see point 4).
}

3) iRZ pulses in PDM-PSK formats were proposed because they quite effectively suppress XPolM [16], [17]. The XPolMNLT curves in a homogeneous WDM system with iRZ-PDMPSK formats are in fact seen to shift to a much higher value than with NRZ pulses. The physical reason can be attributed to the symbol-time periodic alternation of each signal's polarization between two antipodal SOPs, which partly compensates the XPolM polarization spread [16].

4) PSK formats with iRZ pulses are also less sensitive to XPM due to a more constant transmitted PDM power than with NRZ pulses. This translates into weaker intensity-dependent scalar nonlinearities, namely, SPM and XPM [40].

In the DM link, for $\mathrm{iRZ}$ pulses we note an anomalous decrease with $R$ of the initially very high noiseless XPM-NLT. This is ascribed to an increase with $R$ of the XPM-enhancing intensity fluctuations induced by GVD on the high-power, narrow RZ pulses, which overcome the XPM-suppressing action of walkoff. However, the realistic noisy XPM-NLTs are much lower and do recover an increasing behavior with $R$.

5) with iRZ-PDM-PSK formats, the suppression of XPolM makes noisy XPM no longer negligible. Also, the noise loading method may be quite inaccurate on the overall WDM-NLT, with a maximum difference of $2 \mathrm{~dB}$ between noisy and noiseless propagation over the shown $R$ range.

6) SPM-NLT decreases at increasing symbol rates for all formats. SPM manifests at small $R$ as a quasi-periodic nonlinear phase rotation induced by the quasi-periodic signal intensity, which is effectively suppressed by the CPE block, hence SPMNLT is initially large. As the symbol rate increases, the SPM perturbation field evolves into an almost signal-independent circular noise, much like in DU links [33], and the CPE block cannot suppress the intensity fluctuations of the SPM field. Noisy SPM manifests at small $R$ as an ASE-induced nonlinear phase noise: ASE destroys the noiseless-SPM periodicity so that the CPE is less effective in suppressing SPM (Cfr point 1 for XPM). With standard SMFs, the nonlinear signal-noise interactions are limited to bandwidths not exceeding a few tens of $\mathrm{GHz}$ [32], so that, as the symbol-rate increases, a larger and larger fraction of ASE is white as in linear propagation and thus noise loading simulations yield the same results as with distributed noise. Hence noisy and noiseless SPM-NLTs merge at large $R$.

In NRZ-BPSK, the plateau at which the noiseless SPM-NLT levels-off as $R$ is decreased is due to the large powers tolerated by this format. Such powers cause a strong SPM-induced bandwidth enlargement, and a significant fraction of the signal energy is cut off by the receiver filter.

7) the WDM-NLT of PS-QPSK is almost $1 \mathrm{~dB}$ higher than that of NRZ-PDM-QPSK, but still lower than that of NRZ-PDMBPSK. With only XPolM, PS-QPSK has a NLT very close to PDM-BPSK, since they both display a binary SOP pattern on the Poincar sphere (Cfr point 3)). With only XPM, PS-QPSK has a similar NLT to PDM-QPSK since they both have a similar intensity profile and the same quaternary modulation in each polarization.

8) signal-noise interactions in 16QAM are a second-order effect, not only for XPolM, but also for scalar SPM and XPM, because of the large modulation-induced intensity fluctuations of the QAM format. The same reason makes XPM dominant at low symbol rates. 

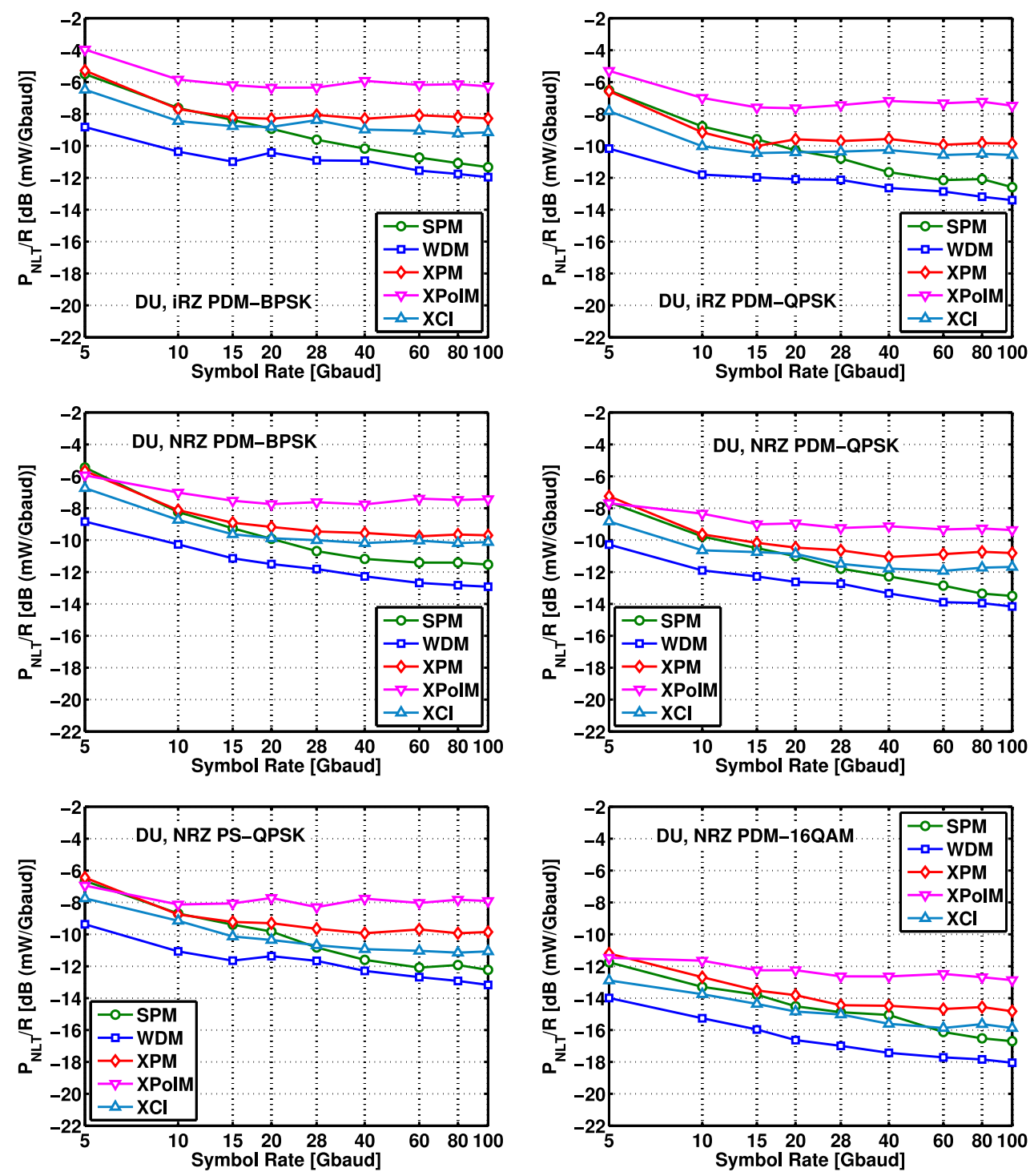

Fig. 4. Normalized margin $10 \log _{10}\left(P_{\mathrm{NLT}} / R\right)$ versus symbol rate $R$ [log scale] for a 15 -channel homogeneous WDM comb with $R / \Delta f=0.56$ over a $20 \times 100$ km SMF DU link. ASE loading at the receiver side. Labels: "SPM" = self-phase modulation only; "XPM" = scalar cross-phase modulation only; "XPolM" = cross-polarization modulation only; "WDM" = all nonlinearities; "XCl" = XPM+XPolM.

9) the decreasing trend of SPM with $R$ leads to a cross point where single-channel NLI dominates over cross-channel NLI. This point, for a 15-channel transmission, is placed around 100 Gbaud for NRZ-BPSK format, while it lies in the range 40-60 Gbaud for the other NRZ formats; it is around 25 Gbaud for the iRZ-PSK formats.

10) The overall WDM-NLT is roughly constant (within $1.5 \mathrm{~dB}$ ) over the shown $R$ range, with a very shallow maximum in the range 10-28 Gbaud. It is worth noting that the influence of signal-ASE interactions on the WDM-NLT is significant only for the iRZ-PSK formats. This implies that NLT at $\mathrm{BER}=10^{-3}$ in WDM DM links for all other formats can be assessed by using the faster noise-loading method.

\section{B. DU Link}

Fig. 4 show the corresponding normalized margin $P_{\mathrm{NLT}} / R$ versus symbol rate in the DU link. Here ASE was loaded at the receiver since signal-noise interactions are negligible [10]. These six plots show that:

11) with all formats, scalar XPM slightly dominates over XPolM. We ascribe this behavior to the GVD-induced pseudorandom intensity variations in DU links, which both tend to enhance XPM variance and weaken XPM-induced symbol correlations observed in the DM case, thus reducing the CPE XPM suppression effectiveness. However, both XPM and XPolM are much better suppressed than in DM links by the huge walk-off of the line;

12) in the presented 15-channel WDM system, the XPM- and XPolM-NLTs are quite close, hence to better compare crossand single-channel NLTs it is useful to also visualize the XCINLT where both effects are active. We see that at lower $R$ $\mathrm{XCI}$ dominates, while at larger $R$ SPM dominates. The crosspoint is around 20 Gbaud for most formats, except for 16QAM where it is around 40-60 Gbaud. When nonlinearity $i$ is active, with $i \in\{\mathrm{XPM}, \mathrm{XPolM}\}$, for all links where it manifests as a 
circular complex Gaussian field, the NLT $P_{\mathrm{NLT}, i}$ is related to the corresponding NLI coefficient $a_{i}$ (see Appendix A) as [13, p. B211]:

$$
P_{\mathrm{NLT}, i}^{2}=1 /\left(3 c^{2} S_{0} a_{i}\right)
$$

where $c=1.27$ for the NLT at $1 \mathrm{~dB}$ penalty, and $S_{0}$ is the electrical SNR at the reference BER. If XPM and XPolM fields were independent, their variances would add up, hence $a_{\mathrm{XCI}} \cong$ $a_{\mathrm{XPM}}+a_{\mathrm{XPolM}}$ and we would get an XCI-NLT:

$$
P_{\mathrm{NLT}, \mathrm{XCI}}=1 / \sqrt{1 / P_{\mathrm{NLT}, \mathrm{XPM}}^{2}+1 / P_{\mathrm{NLT}, \mathrm{XPolM}}^{2}} \text {. }
$$

We verified that such a formula is very close (within $0.3 \mathrm{~dB}$ for all formats, except for PS-QPSK where the error can be up to $1 \mathrm{~dB}$ ) to the XCI simulated NLT in Fig. 4, hence we conclude that XPM and XPolM are practically uncorrelated in DU links for most formats. We also verified that (2) very accurately predicts the XCI-NLT even in DM links.

13) the GN reference formula [19, eq. (1)] treats the crossnonlinearity as a whole, and cannot discriminate between XPM and XPolM. At large $R$, the XCI coefficient is predicted to scale as [41, eq. (20)] ${ }^{4}: a_{\mathrm{XCI}} \propto 1 /(R \Delta f)=\eta / R^{2}$ (consistent with [43, eq. (57)] for Nyquist-WDM systems). Using (1) we have $P_{\mathrm{NLT}, \mathrm{XCI}} \propto 1 / \sqrt{a_{\mathrm{XCI}}}$, hence the GN model leads us to conclude that $P_{\mathrm{NLT}, \mathrm{XCI}} / R$ is a constant at large $R$ and constant $\eta$. This confirms the observed flattening of the simulated XCINLTs versus $R$ for all formats.

14) $X P$ PolM is much weaker than in the DM case. In both DM and DU links, XPolM operates in the same way within each span, with an efficiency related to the number of pivot re-orientations along propagation [30]. The difference is that the periodic map of the DM link induces a long trajectory of the output SOP vector over the Poincar sphere. In the DU link, instead, the direction of the pivot is almost uncorrelated span by span, thus producing Brownian-like wiggled output SOP trajectories that drift less away from the input SOP [30]. Since each symbol experiences a different SOP trajectory, overall we observe polarization scattering, and since the drift from the input SOP is smaller in DU links, then we also have a weaker XPolM.

15) with all formats, the WDM $P_{\mathrm{NLT}} / R$ curves decrease with $R$, i.e., the best symbol rate at fixed bandwidth efficiency and with 15 channels is below the lower $R$ limit, i.e., 5 Gbaud.

\section{More Checks with GN Theory}

NLT simulations are extremely time-consuming hence the number of WDM channels was here limited to 15 . It would be very useful to have an analytical model to cross-verify the general physical trends found by simulation and to extrapolate the behavior when the WDM channel count is in the order of 100 , as in typical commercial WDM systems. Fortunately, for DU systems the GN model [19] does the job. In this section, we test the predictions of the GN model and compare them with those obtained from the NLT data in Figs. 3 and 4.

If the NLI coefficient $a_{i}$ for the $i$ th nonlinearity were formatindependent, as postulated by the GN model, from (1) we would

\footnotetext{
${ }^{4}$ There is a typo in eq. (20) of [41], the correct equation can be found in [42].
}

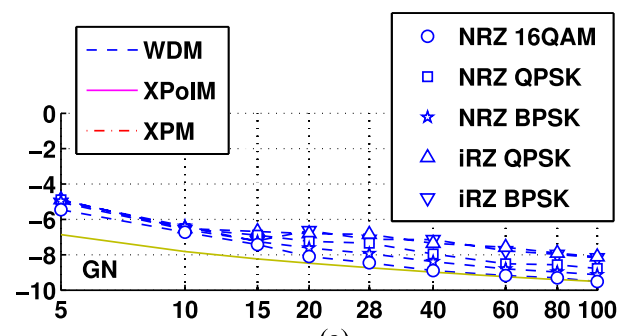

(a)

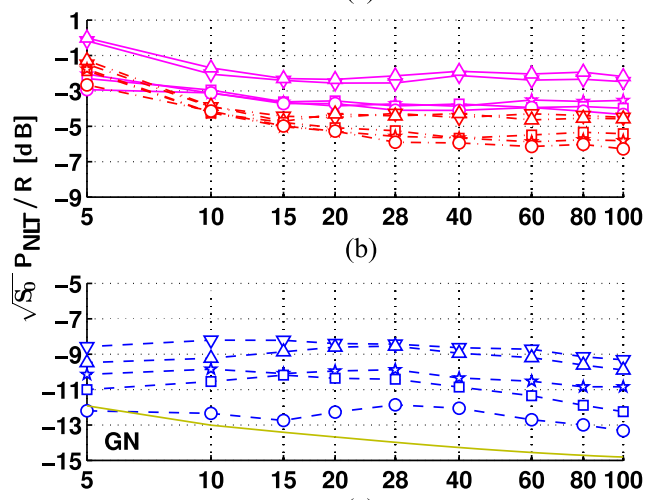

(c)

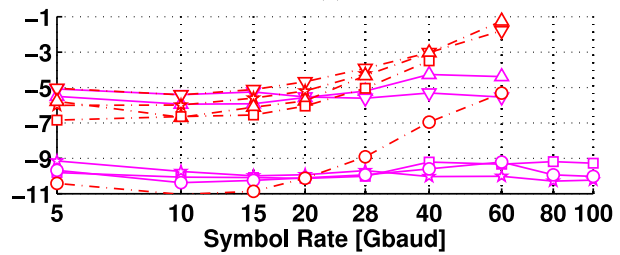

(d)

Fig. 5. Lines with markers: simulated $\sqrt{S_{0}} P_{\mathrm{NLT}} / R$ [dB(mW/Gbaud)] versus $R$ [Gbaud] for DU(a-b) and DM(c-d) $20 \times 100 \mathrm{~km} \mathrm{SMF} \mathrm{link} \mathrm{with} 15 \mathrm{WDM}$ channels at $\eta=0.56$, (Dashed) WDM, (Solid) XPolM, (Dash-dotted) XPM. $P_{\mathrm{NLT}} / R$ simulated values are those reported in Figs. 3 and 4 . GN theory plotted in solid line without markers as $\sqrt{S_{0}} P_{\mathrm{NLT}} / R=1 / \sqrt{3 c^{2} a_{\mathrm{NL}}} / R$.

expect $\sqrt{S_{0}} P_{\mathrm{NLT}, i}$ to also be format-independent. If this were the case, the simulated $P_{\mathrm{NLT}, i} / R$ versus $R$ curves reported in Fig. 4 for the DU link, multiplied by the $\sqrt{S_{0}}$ of the used format, should all fall on top of each other. We performed this check in Fig. 5(a) and (b). We used the same $S_{0}$ values as in the simulations, namely, $S_{0}=[7.77,10.77,17.07] \mathrm{dB}$ for the three NRZ formats (BPSK, QPSK, 16QAM in that order), while iRZ formats required $0.2 \mathrm{~dB}$ less SNR than their corresponding NRZ formats. Dashed curves refer to XPolM, dash-dotted to XPM, and solid lines to the all-nonlinearities WDM case. We note that the $\sqrt{S_{0}} P_{\mathrm{NLT}} / R$ XPM/XPolM curves reasonably merge, with different values between NRZ and iRZ curves. The WDM curves with NRZ pulses show instead a spread of about $1.5 \mathrm{~dB}$ across the formats at larger $R$, which we verified to be also due to the spread of the corresponding SPM curves (not shown to avoid clogging the figure). As pointed out in Section II, $P_{\mathrm{NLT}} / R$ is proportional to the reach, hence one can read off the WDM curves in Fig. 5(a) also the reach behavior versus symbol rate for each modulation format. A similar decreasing reach versus $R$ behavior for a five-channel WDM DU system was reported in [7].

We also report in Fig. 5(a)-(c) the solid-line curve labeled GN, which plots the theoretically equivalent quantity $1 / \sqrt{3 c^{2} a_{\mathrm{NL}} R^{2}}$, as per eq. (1), with $a_{\mathrm{NL}}=\int G_{\mathrm{NLI}}(f) \mathrm{d} f$ 


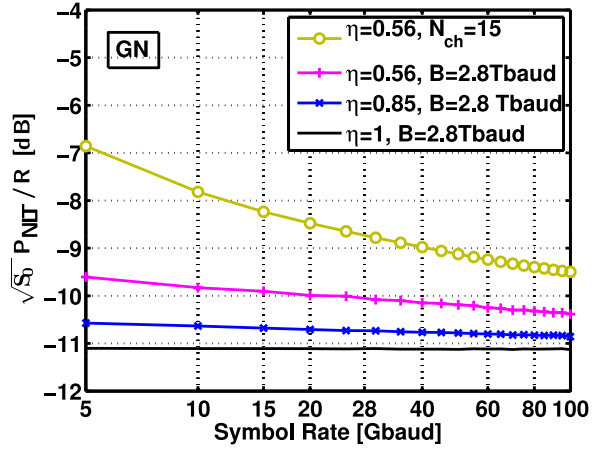

Fig. 6. Plot of $\sqrt{S_{0}} P_{\mathrm{NLT}} / R$ versus $R$ for DU $20 \times 100 \mathrm{~km}$ SMF link obtained from GN reference formula at varying bandwidth efficiency $\eta$ and fixed aggregate symbol rate $B=N_{\mathrm{ch}} R$. The $\eta=0.56, N_{\mathrm{ch}}=15$ curve is the same labeled GN in Fig. 5(top). These curves, which according to the GN model are format-independent, display the same behavior versus $R$ as the reach, as proved in Appendix A.

calculated according to the GN reference formula [19, eq. (1)] with the efficient algorithm detailed in[20], with the same normalized WDM input power spectral density $G_{\mathrm{NLI}}$ used in the simulations of Fig. 4. The GN curve theoretically confirms the monotone decreasing trend versus $R$ in DU links with $\eta=0.56$ and 15 channels. Among the NRZ formats, we note that the 16QAM format is the closest to the GN curve, because of its better agreement with the key GN model assumption of a Gaussian input process [20]. Such findings about a non-negligible modulation-format dependence of the NLI variance are in agreement with recent extensions of the GN model [20]-[23]. Also, note that iRZ formats are the farthest from the GN model, with a distance of over $2 \mathrm{~dB}$, and their weaker NLI may suggest that iRZ formats do not converge to a Gaussian-like field as fast as their NRZ counterparts.

In Fig. 5(c)-(d) we tried the same rescaling with the DM link data in Fig. 3, where we used the distributed-noise NLTs only. We see that the XPM curves now show a more marked format dependence (note the dramatic decrease of the 16QAM XPM), while the XPolM curves for the NRZ formats do reasonably merge together and are relatively flat, as in the DU link. This is an indication that XPolM is the nonlinear effect that behaves most similarly in DM and DU links. The large spread of WDM curves across the modulation formats now comes not only from a large spread of the SPM curves (not reported to avoid confusion), but also from that of the XPM curves. For completeness, the curve labeled GN reports the predictions of the GN model for the DM link, which are clearly unsatisfactory. A recent extended GN model seems promising in reducing such a spread also for DM links [44].

To conclude this section, we present GN model extrapolations of the $\sqrt{S_{0}} P_{\mathrm{NLT}} / R$ versus $R$ curves to large WDM channel numbers, where simulations are unfeasible. We first note that the monotonically decreasing behavior versus $R$ of both GN theory and simulations observed in Fig. 5(a) can in part be explained by the increase of the total WDM bandwidth, since the number of channels was fixed at a fixed $\eta=R / \Delta f=0.56$, hence by increasing $R$ we also increased the spacing $\Delta f$. In Fig. 6 we now more realistically fixed the WDM aggregate symbol rate $B=N_{\text {ch }} R=2.8$ Tbaud, which corresponds to increasing the
TABLE I

$\Delta=P_{\mathrm{NLT}}\left(N_{\mathrm{ch}}=15\right)-P_{\mathrm{NLT}}\left(B_{\mathrm{A}}\right)[\mathrm{dB}]$ COMPUTED FROM WDM $P_{\mathrm{NLT}}$ AT REFERENCE BER $=10^{-3}$ OVER A $20 \times 100 \mathrm{KM} \mathrm{SMF} \mathrm{LINK}$

\begin{tabular}{llllll}
\hline \hline & \multicolumn{2}{c}{$\eta=0.56\left(B_{\mathrm{A}}=750 \mathrm{GHz}\right)$} & & $\eta=0.85\left(B_{\mathrm{A}}=500 \mathrm{GHz}\right)$ \\
\cline { 2 - 3 } \cline { 5 - 6 } & 10 Gbaud & $80 \mathrm{Gbaud}$ & & $10 \mathrm{Gbaud}$ & $80 \mathrm{Gbaud}$ \\
\hline 16QAM, DM & $-0.44 \mathrm{~dB}$ & $0.53 \mathrm{~dB}$ & & $-0.30 \mathrm{~dB}$ & $0.40 \mathrm{~dB}$ \\
16QAM, DU & $-0.49 \mathrm{~dB}$ & $0.61 \mathrm{~dB}$ & & $-1.18 \mathrm{~dB}$ & $0.37 \mathrm{~dB}$ \\
QPSK, DM & $-0.40 \mathrm{~dB}$ & $0.65 \mathrm{~dB}$ & & $-0.57 \mathrm{~dB}$ & $0.73 \mathrm{~dB}$ \\
QPSK, DU & $-0.25 \mathrm{~dB}$ & $0.42 \mathrm{~dB}$ & & $-0.76 \mathrm{~dB}$ & $0.73 \mathrm{~dB}$ \\
\hline
\end{tabular}

number of channels $N_{\text {ch }}$ when reducing $R$. The total occupied bandwidth was fixed at $W=B / \eta$. We observe that even in this case the $\sqrt{S_{0}} P_{\mathrm{NLT}} / R$ (hence the reach!) plot versus $R$ keeps a monotonically decreasing trend at any $\eta<1$, and converges to the naturally flat behavior of the Nyquist-WDM case $(\eta=1)$. The physical message from these curves is that, for reach maximization, smaller symbol rates are slightly preferable, which means the WDM energy should be most uniformly spread across the frequency axis. However, from the "reach" curves of Fig. 6, we conclude that the granularity of the WDM spectrum may be of some importance only away from the Nyquist limit, and at small channel counts, where smaller symbol rates are slightly preferable. Otherwise, in all practical cases, the per-channel symbol rate need not be optimized to maximize the reach. We cannot replicate such curves by SSFM simulation. However, to give a feeling to the reader, for NRZ QPSK and 16QAM we replicated at $R=10$ and 80 Gbaud the WDM NLTs of Figs. 3, 4, and 7 at the aggregate bandwidth $B_{\mathrm{A}}$ of the 15-channel system at 28 Gbaud namely, $B_{\mathrm{A}}=750 \mathrm{GHz}$ at $\eta=0.56$. We also included the case $\eta=0.85\left(B_{\mathrm{A}}=500 \mathrm{GHz}\right)$ that will be analyzed in Section IV-D. Table I shows the NLT gap of the new curves from the corresponding ones in Fig. 3, 4, and 7. From the table we note a counterclockwise tilt of each curve. This was expected, since we are enhancing cross-nonlinearities at $10 \mathrm{Gbaud}$ while reducing them at 80 Gbaud. Regarding the DU case such results qualitatively agree with the GN model predictions of Fig. 6. Overall we observe that for the tested symbol rates the NLTs change by at most $1.18 \mathrm{~dB}$, and a shallow maximum of the reach at 5 Gbaud for DU links in reasonable agreement with [1], [9].

\section{NLT at Large Bandwidth Efficiency}

Fig. 7 shows $P_{\text {NLT }} / R$ versus symbol rate for an NRZ PDMQPSK signal in both a DM link (top) and a DU link (bottom), with a larger bandwidth efficiency $\eta=0.85$ w.r.t. that of Fig. 3 . Comparing the figure with the corresponding plots in Fig. 3, (second row, left) and (fourth row, left), we observe that an increase of $\eta$ (hence a reduction of $\Delta f$ at same $R$ and thus a decrease of the walk-off) enhances cross-channel effects. In the DM link, XPolM remains the dominant nonlinearity, with a NLT decrease from the $\eta=0.56$ case by about $1-1.5 \mathrm{~dB}$ at $R>28$ Gbaud. Overall, the WDM curve shifts downwards by more than $1 \mathrm{~dB}$, and keeps its concave shape with a shallow maximum in the 10-40 Gbaud range, with signal-ASE interactions still negligible over the whole symbol rate range. Even if not reported here, we observed similar NLT reductions even in the 16QAM 

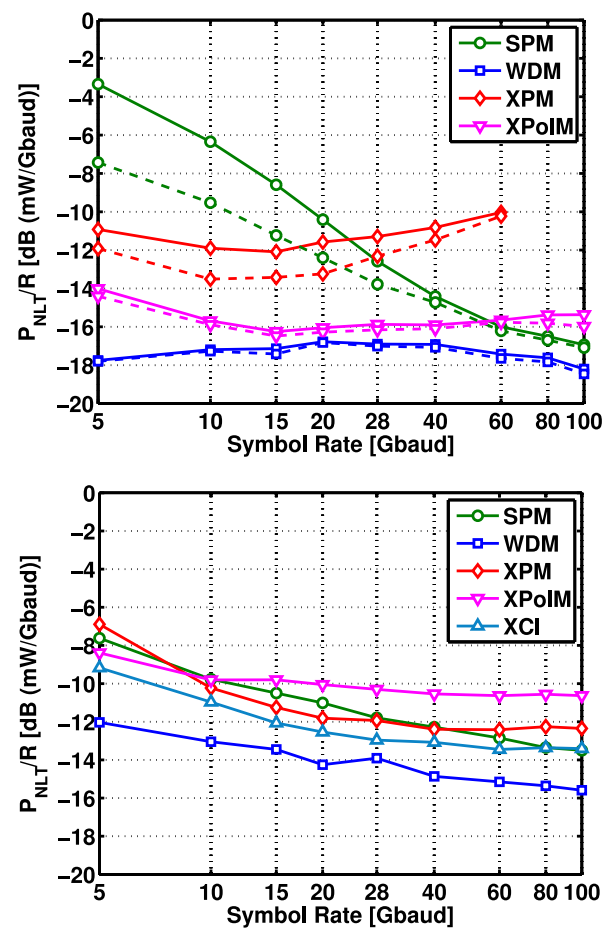

Fig. 7. Normalized margin $P_{\mathrm{NLT}} / R$ at reference BER $=10^{-3}$ versus symbol rate for 15-channel NRZ-PDM-QPSK homogeneous WDM comb with $\eta=0.85$ over a $20 \times 100 \mathrm{~km}$ SMF link. (Top) DM link, (Bottom) DU link.

case. Hence, the qualitative aspects observed in Sections IV-A and IV-B still remain true at $\eta=0.85$.

In the DU link, we observe that both XPM- and XPolM-NLTs at large rates decrease by $\sim 1 \mathrm{~dB}$ when increasing $\eta$ by $\sim 2 \mathrm{~dB}$, and so does the WDM-NLT curve. This is consistent with the GN model large-rate prediction that $a_{\mathrm{XCI}} \propto 1 /(R \Delta f)=\eta / R^{2}$ [41]. Using (1) we have $P_{\mathrm{NLT}, \mathrm{XCI}}^{\mathrm{dB}} \propto-\frac{1}{2} \eta^{\mathrm{dB}}$. Now XPM-NLT is quite close to the SPM-NLT, while the XPolM-NLT is almost $2 \mathrm{~dB}$ larger than the XPM-NLT around 60 Gbaud.

At lower rates, FWM due to next neighbors starts to be noticeable even in DU links. For instance, at $R=5$ Gbaud the XPM- and XPolM-NLTs at $\eta=0.85$, vary very little w.r.t. the $\eta=0.56$ case; however, the WDM-NLT (which captures FWM) decreases by $1.8 \mathrm{~dB}$. To double-check that next-neighbor FWM is indeed the cause, in a separate test of the same link we suppressed the two next neighbors of the central channel in the 15 channel comb and verified that the true WDM-NLT now coincides (to within $0.5 \mathrm{~dB}$ ) with the SFP-obtained NLT (see Appendix B) with XPM + XPolM + SPM activated.

\section{REACH PREDICTION FROM NLT}

The procedure to obtain the 1-dB NLT $P_{\mathrm{NLT}}$ consists of tuning the lumped amplifiers noise figure together with the launch power in order to achieve the combination such that at an SNR $S=S_{0}+1 \mathrm{~dB}$ the BER is $10^{-3}$ [32]. As a by-product of the $P_{\mathrm{NLT}}(N)$ estimation for an $N$-span link, we thus also get the corresponding EDFA's noise figure $F_{\mathrm{NLT}}(N)$. Table II reports the values of $F_{\mathrm{NLT}}$ at $N=20$ spans found in the same WDMNLT simulations yielding the $P_{\mathrm{NLT}}$ values used to build Fig. 3 for three selected modulation formats at $R=10,28$ and 80
TABLE II

EDFA's Noise Figure $F_{\text {NLT }}$ [dB] Yielding 1-dB of SNR PENALTY AT BER $=10^{-3}$ AND POWER $P_{\text {NLT }}$ Estimated In Figs. 3-4

\begin{tabular}{lccc}
\hline \hline & 10 Gbaud & 28 Gbaud & 80 Gbaud \\
\hline DM, 16QAM & -2.90 & -2.32 & -3.55 \\
DU, 16QAM & 2.68 & 0.95 & -0.38 \\
DM, QPSK & 9.17 & 9.48 & 7.60 \\
DU, QPSK & 12.69 & 11.86 & 10.35 \\
DM, BPSK & 14.42 & 14.52 & 13.41 \\
DU, BPSK & 17.42 & 15.88 & 14.83 \\
\hline \hline
\end{tabular}

$20 \times 100 \mathrm{~km}$ SMF link with 15 WDM channels. ASE noise loaded at the receiver.

Gbaud in both DM and DU links, with receiver noise loading (since Fig. 3 indicates that, for NRZ pulses, signal-noise interactions are negligible). Whenever $F_{\mathrm{NLT}}$ takes unrealistic values, it means that a practical system is not able to achieve the target BER at the selected distance. For instance, PDM-16QAM achieved NLT at $N=20$ spans with a negative noise figure, which physically means that 20 spans are unachievable with lumped EDFA amplification and does require distributed amplification. $F_{\mathrm{NLT}}$ however can be used to estimate the system reach $N_{0}$ at a practical noise figure $F$ from NLT measurements over a much shorter $N$-span link by the following formula (derived in Appendix A):

$$
\frac{N_{0}(F)}{N}=\left(\frac{F_{\mathrm{NLT}}(N)}{x_{1} \cdot F}\right)^{\frac{2}{3+\epsilon}}
$$

with $x_{1}=0.94$ and $\epsilon$ is the NLI slope parameter. Of course one also needs the knowledge of $\epsilon$ to perform such an estimation. $\epsilon$ can be obtained either by theoretical models (e.g., the GN model predicts $\epsilon \sim 0$ when cross-channel nonlinearity dominates [19], [41]) or by numerical fitting.

To double-check the accuracy of formula (3) for both our DU and DM links, we performed extra UFP SSFM simulations for three NRZ modulation formats (PDM-BPSK, PDM-QPSK and PDM-16QAM) at three sample symbol rates $(R=10,28$ and 80 Gbaud). Fig. 8 plots the power yielding the reference $\mathrm{BER}=10^{-3}$ versus number of spans $N$ for a 15 -channel homogeneous WDM comb for the selected modulation formats and symbol rates. The noise figure (reported in each plot) was arbitrarily set to keep the reach between 15 and 40 spans in all cases, and receiver noise loading was used. Triangles show simulations, while solid lines are least-squares cubic interpolations of the simulated values, according to formula [13, eq. (8)], where $a_{\mathrm{NL}}=\alpha_{\mathrm{NL}} N^{1+\epsilon}$, whose only fitting parameters are $\alpha_{\mathrm{NL}}$ and $\epsilon$. From such "contours" the system reach can be easily visualized. The least-squares fitting NLI slope parameter values are reported in Table III, along with the much smaller GN model predictions for $\epsilon$ in DU links[19, Section XI-D]. For our 15-channel DM and DU links we note that $\epsilon$ is very slowly decreasing with symbol rate $R$, and can be safely considered as a constant for reach maximization w.r.t. symbol rate, as discussed in Section II and in Appendix A. For DM links, it is $\epsilon \sim 0.7$ for most formats, except for BPSK which has $\epsilon \sim 0.6$; for DU links the value is much smaller, as discussed in [13], and close to 0.3 , except for QAM which shows an $\epsilon<0.2$. For the 

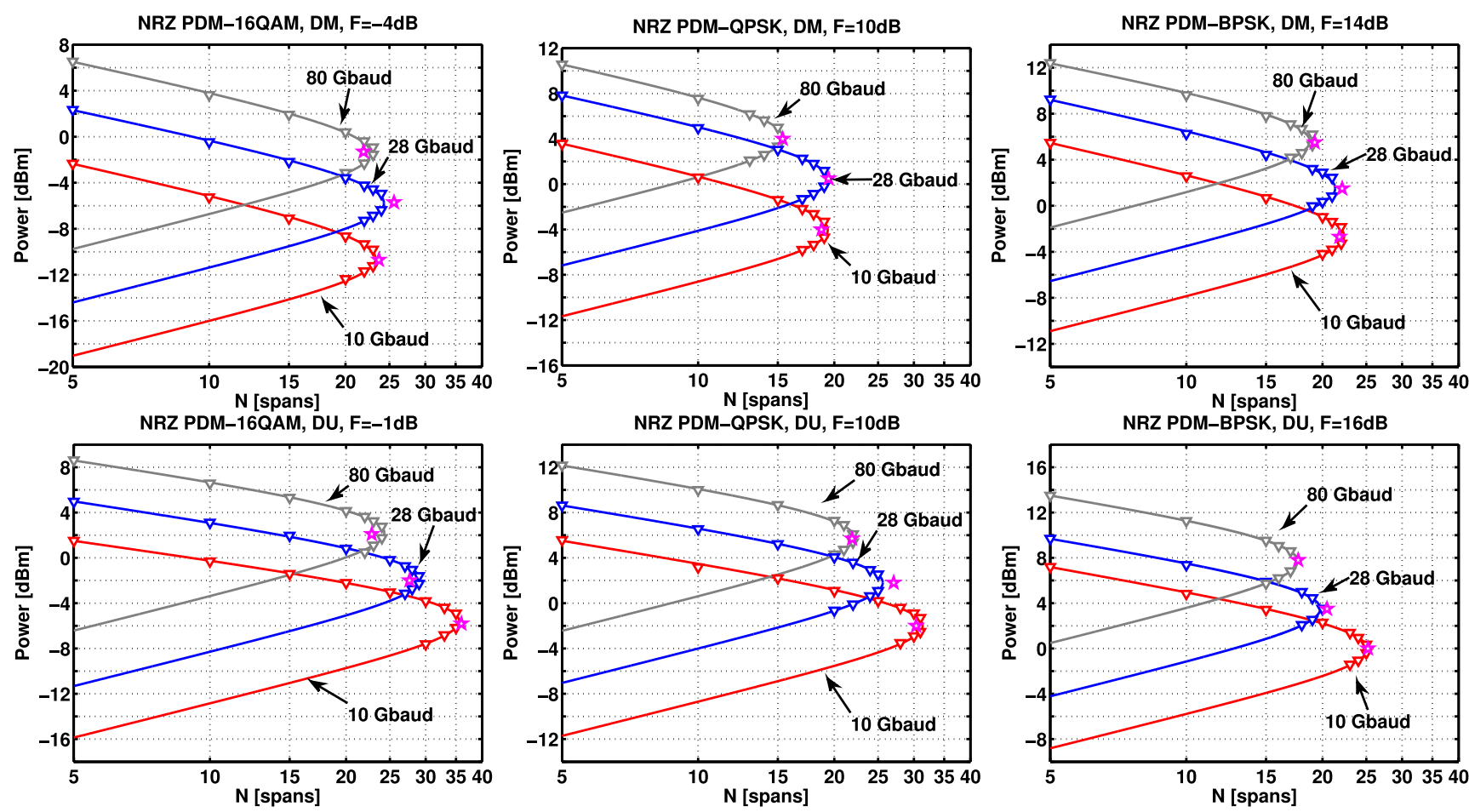

Fig. 8. Triangles: SSFM-simulated power versus span number $N\left(\log _{10}\right.$ scale) at reference BER $=10^{-3}$ for a 15-channel homogeneous WDM comb with $\eta=R / \Delta f=0.56$ over a $N \times 100 \mathrm{~km}$ SMF link. ASE noise loaded at the receiver. (Top row) DM link, (Bottom row) DU link. (Left column) PDM-16QAM, (Center column) PDM-QPSK, (Right column) PDM-BPSK. Stars: prediction by (3) using the NLTs of Fig. 3. Solid lines: least-squares cubic interpolations of triangles.

TABLE III

ESTIMATED $\epsilon$ FROM SIMULATED DATA IN FIG. 8

\begin{tabular}{lccc}
\hline \hline & 10 Gbaud & 28 Gbaud & 80 Gbaud \\
\hline DM, 16QAM & 0.77 & 0.68 & 0.71 \\
DU, 16QAM & 0.11 & 0.19 & 0.19 \\
DM, QPSK & 0.80 & 0.72 & 0.71 \\
DU, QPSK & 0.29 & 0.27 & 0.26 \\
DM, BPSK & 0.77 & 0.70 & 0.56 \\
DU, BPSK & 0.40 & 0.30 & 0.29 \\
GN model [19, Section XI-D] & 0.072 & 0.013 & 0.005 \\
\hline \hline
\end{tabular}

PDM-QPSK DU link, the values in Table III are consistent with the experimental measurements in $[26]^{5}$.

The predictions of formula (3) can now be calculated using Tables III and II and compared to the true reach. They are marked with stars in Fig. 8 and are quite close to the actual reach, with a worst-case error of only 1.5 spans. Although the used noise figures may be unrealistic and were used to keep the reach within 15-35 spans, yet the accuracy of (3) is not expected to degrade with practical noise figures and longer reaches, since such an equation is accurate for all physical links for which the NLT scales as $N^{-(1+\epsilon) / 2}$, with $\epsilon$ a suitably estimated factor. The fitting factor $x_{1}$ is exact if the NLI is Gaussian additive, and may change for different NLI statistics. As a sanity check, we also verified an excellent accuracy of (3) at $B_{\mathrm{A}}=750 \mathrm{GHz}$ (43 channels) for 10 Gbaud 16QAM in both DM and DU links.

${ }^{5}$ For larger WDM systems where cross-nonlinearity dominates the NLT, it is theoretically predicted by the GN model that $\epsilon \sim 0$ [19], [41].

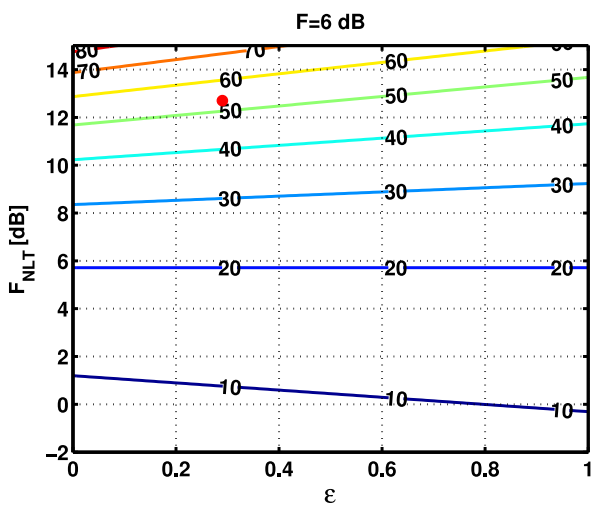

Fig. 9. Contour levels of the maximum reach $N_{0}$ given in (3) versus $\epsilon$ and $F_{\mathrm{NLT}}[\mathrm{dB}]$ at a fixed $F=6 \mathrm{~dB}$. Red circle corresponds to the pair $\left(F_{\mathrm{NLT}}, \epsilon\right)$ estimated in the 10 Gbaud DU-QPSK link (see Tables II and III).

\section{A. Tolerance to Estimation Errors}

In Fig. 8 we showed that with the knowledge of $F_{\mathrm{NLT}}$ and $\epsilon$ it is possible to estimate the system reach with a very good accuracy in a wide range of symbol rates and modulation formats. $F_{\text {NLT }}$ comes for free from NLT simulations at a fixed distance $N$. Knowledge of the NLI slope parameter $\epsilon$ must instead be acquired from several BER measurements versus distance, as those in Fig. 8, whose fit lead to Table III. Alternatively, one can resort to analytical approximate expressions of $\epsilon$. The general question is thus how accurately we need to know $\epsilon$ and $F_{\mathrm{NLT}}$. To answer this question, in Fig. 9 we plotted the contour levels of $N_{0}\left(F_{\mathrm{NLT}}, \epsilon\right)$ obtained from (3) versus both $\epsilon$ and $F_{\mathrm{NLT}}$ at fixed $F=6 \mathrm{~dB}$. Such contours can be used to quantify the tolerance 
to our allowed ignorance of the two key parameters. We note that, according to (3), when the ratio $F_{\mathrm{NLT}} /\left(x_{1} \cdot F\right)=1$ the parameter $\epsilon$ has no impact on reach prediction. A good estimation of $F_{\mathrm{NLT}}$ is more important than a good one of $\epsilon$ as a direct consequence of the reach dependence on the term $3+\epsilon$, where the term 3 smooths out the impact of the small $\epsilon$. For instance, imagine to estimate the reach of the 10 Gbaud DU-QPSK link at $F=6 \mathrm{~dB}$ using equation (3). To this aim we need $F_{\mathrm{NLT}}$ and $\epsilon$, which are provided by Tables II and III. By inserting such values in (3) we get a reach of $N_{0}=53$ spans. To get a feeling of the uncertainty of this value, we report in Fig. 9 by a red circle the pair $\left(F_{\mathrm{NLT}}, \epsilon\right)$ estimated for this system. By looking at the contour levels we infer that an error of $1 \mathrm{~dB}$ on $F_{\mathrm{NLT}}$ gives a sizable error of seven spans in reach, while a significant error of $22 \%$ on $\epsilon$ yields an error of just one span in reach.

\section{CONCLUSION}

This paper provided simulation estimations of the NLT $P_{\mathrm{NLT}}$ versus channel symbol rate $R$ for both DM and DU coherent systems and for several modulation formats and supporting pulse shapes of interest. By using the nonlinearity-decoupling method detailed in Appendix B, we provided the $P_{\mathrm{NLT}}$ due to each individual Kerr effect, and showed that one can gather valuable information about the dominant nonlinear effect as the rate $R$ is varied. We provided plausible explanations of the observed behavior based on the main existing analytical nonlinear models. We proved that plots of $P_{\mathrm{NLT}} / R$ also show the symbol rate that maximizes the transmission distance, i.e., the reach. For DU systems, for which the rather accurate analytical GN model is available, we compared our NLT simulations against theory and provided theoretical extrapolations to practically large WDM systems which cannot be simulated. We finally provided a new, simple system-level formula for reach prediction that uses the noise figure obtained at NLT, and we showed that such a formula can be used for accurate reach predictions both in DU and DM systems, provided that estimations of the NLI accumulation parameter $\epsilon$ are available.

\section{APPENDIX A \\ GN Model ReACH PREDICTION IN DU LINKS}

Recent work provided a model to justify the performance of coherent DU links [19], [43]. The model assumes the NLI is a stationary, signal-independent circular GN, like the ASE noise, whence the GN name. In this framework, the received SNR over the electrical receiver bandwidth is [36], [37], [45]:

$$
S=\frac{P}{N_{\mathrm{ASE}}+a_{\mathrm{NL}} P^{3}}
$$

where $P$ is the power per channel, $N_{\mathrm{ASE}}$ is the ASE power, and $a_{\mathrm{NL}}$ is the NLI coefficient. Assuming matched-filter detection, the BER can be expressed as BER $=f(S)$, where the functional relation depends on the modulation format. The model provides useful insights especially when working at a target BER, i.e., a target $\mathrm{SNR}^{6} S_{0}$. In this constrained scenario, optimizing performance means maximizing the transmission distance, i.e., the

\footnotetext{
${ }^{6}$ with matched filter detection, $S_{0}$ coincides with $\mathcal{E}_{s} / \mathcal{N}_{0}$, being $\mathcal{E}_{s}$ the average energy per symbol and $\mathcal{N}_{0}$ the one-sided noise power spectral density.
}

number of spans. Equation (4) is the basic ingredient in the maximization problem. To proceed, one further needs to know how the ASE and the NLI scale with span number $N$. While $N_{\mathrm{ASE}}=\beta N$ is clearly linear in $N$ with factor $\beta=h \nu F G R$ ( $h \nu$ : photon energy at frequency $\nu ; F$ : amplifiers noise figure; $G$ : amplifiers gain; $R$ : matched-filter electrical bandwidth, equal to symbol rate), in [19], [26], and [13] it was shown that the NLI coefficient scales approximately as $a_{\mathrm{NL}}=\alpha_{\mathrm{NL}} N^{1+\epsilon}$, $0 \leq \epsilon \leq 1$, due to correlated and unequal NLI contributions from each optical span [26].

The idea is now to express the maximum transmission distance (reach) $N_{0}$ corresponding to the parameters pair $\left(\alpha_{\mathrm{NL}}, \epsilon\right)$ by using available NLT measurements at a specific coordinate $N$. We find it convenient to introduce the constrained NLT $\hat{P}_{\mathrm{NLT}}(N)$ as the power maximizing (4), i.e., $\hat{P}_{\mathrm{NLT}}(N)=$ $\arg \max _{P}[S(P, N)]$, and such that $S\left(\hat{P}_{\mathrm{NLT}}, N\right)=S_{0}$. The first requirement implies that at $\hat{P}_{\mathrm{NLT}}$ the ASE power is twice the NLI power [37]. When used together with the second requirement, it turns (4) into the following alternative identities [13]:

$$
S_{0}=\frac{1}{3 \alpha_{\mathrm{NL}} \hat{P}_{\mathrm{NLT}}^{2}(N) \cdot N^{1+\epsilon}}=\frac{\hat{P}_{\mathrm{NLT}}(N)}{\frac{3}{2} \hat{\beta}(N) \cdot N}
$$

where $\hat{\beta}(N) \triangleq h \nu \hat{F}_{\mathrm{NLT}}(N) G R$ and the hat reminds us that to satisfy both requirements at $N \neq N_{0}$ the ASE noise parameter should be tweaked away from its true value $\beta . \hat{\beta}(N)$ is the fictitious ASE parameter than makes $N$ be the reach, hence $\hat{\beta}\left(N_{0}\right) \equiv \beta$. From (5) we then get the following relation, first reported in [13, eq. (24)]:

$$
\frac{N_{0}(F)}{N}=\left(\frac{\hat{P}_{\mathrm{NLT}}(N) / R}{\frac{3}{2} N h v G F S_{0}}\right)^{\frac{2}{3+\epsilon}} .
$$

Eq. (6) is consistent with the reach predictions in [43] when $\epsilon=0$. Since $\epsilon$ is almost independent of $R$, as checked from measurements reported in Table III, from (6) we learn that maximizing the value $\hat{P}_{\mathrm{NLT}}(N) / R$ measured at a generic distance $N$ corresponds to maximizing the system reach $N_{0}$. Hence, as done in the main text, from plots of $\hat{P}_{\mathrm{NLT}} / R$ versus $R$ we can immediately spot the distance-maximizing symbol rate at a fixed bandwidth efficiency.

In this paper, we used the power $P_{\mathrm{NLT}}$ yielding $1 \mathrm{~dB}$ of SNR penalty at BER $=10^{-3}$ w.r.t. linear transmission, instead of $\hat{P}_{\mathrm{NLT}}$ which yields a penalty of $1.76 \mathrm{~dB}$ [13]. It can be proved that $\hat{P}_{\mathrm{NLT}}=c P_{\mathrm{NLT}}, c=1.27$, for any link where the GN model applies [13], e.g., DU SMF links at large-enough symbol rates. Since by definition $N_{0}\left(\hat{F}_{\mathrm{NLT}}(N)\right)=N$, then by multiplying and dividing the fraction on the right-hand side (r.h.s.) of (6) by $\hat{F}_{\mathrm{NLT}}(N)$ and by using the second identity of (5) we get:

$$
\frac{N_{0}(F)}{N}=\left(\frac{\hat{F}_{\mathrm{NLT}}(N)}{F}\right)^{\frac{2}{3+\epsilon}}
$$

which relates the maximum reach obtained at an arbitrary noise figure $F$ to the constrained noise figure $\hat{F}_{\mathrm{NLT}}$ measured at $N$ spans. Eq. (7) is preferable to (6) since it does not involve the precise knowledge of the receiver bandwidth, which is $R$ only 
with matched-filtering but in practice is set by the butterfly equalizer that also implements polarization demultiplexing.

In our simulations, instead of estimating $\hat{F}_{\mathrm{NLT}}$, we estimated $F_{\mathrm{NLT}}$, i.e., the tweaked noise figure at the $1-\mathrm{dB} P_{\mathrm{NLT}}$ at $N$ spans. The ratio $x_{y} \triangleq F_{\mathrm{NLT}, y} / \hat{F}_{\mathrm{NLT}}$ for a penalty of $y[\mathrm{~dB}]$ is plotted in [13, Fig. 6] as a function of $y$. At $y=1 \mathrm{~dB}$ we read off the value $x_{1}=0.94$, hence substitution in (7) leads to eq. (3) in the main text. It can be proved that in general $x_{y}=\frac{3}{2 c(y)}-\frac{1}{2 c(y)^{3}}$, where $c(y)=1 / \sqrt{3\left(1-10^{-y / 10}\right)}$ (hence $\left.c(1)=1.27\right)$.

\section{APPENDIX B}

\section{NONLINEARITY-DECOUPLING METHOD}

In this Appendix we summarize the nonlinearity-decoupling method, first introduced in [18], which we used in SSFM simulations to selectively activate the desired nonlinear Kerr effects in the nonlinear step. For a PDM WDM signal

$$
\vec{A}(z, t)=\sum_{k=1}^{N_{\text {ch }}} \vec{A}_{k}(z, t) \mathrm{e}^{j 2 \pi k \Delta f t}
$$

with channel spacing $\Delta f$ and PDM complex signal envelopes $\vec{A}_{k}(z, t) \triangleq\left[A_{k x}(z, t), A_{k y}(z, t)\right]$, the Manakov nonlinear step for the $n$th channel, $n=1, \ldots, N_{\mathrm{ch}}$, can be expressed in $a b$ sence of FWM by [30]:

$$
\frac{\partial \vec{A}_{n}}{\partial z}=-i \bar{\gamma}\left(\left(A_{n}^{2}+\frac{3}{2} \sum_{k \neq n} A_{k}^{2}\right) \sigma_{0}+\frac{1}{2} \sum_{k \neq n}\left(\vec{a}_{k} \cdot \vec{\sigma}\right)\right) \vec{A}_{n}
$$

where $A_{k}^{2}$ is the signal intensity of channel $k$ at time $t$, $\bar{\gamma}=(8 / 9) \gamma$ is the Manakov-averaged nonlinear coefficient, $\sigma_{0}$ is the $2 \times 2$ identity matrix, $\vec{\sigma}$ is the Pauli spin vector [46], $\vec{a}_{k}=\vec{A}_{k}^{\dagger} \vec{\sigma} \vec{A}_{k}$ is the real 3-D Stokes vector associated with the complex Jones vector $\vec{A}_{k}$, and $\vec{a}_{k} \cdot \vec{\sigma}=\sum_{i} a_{k i} \sigma_{i}$ is a (generally non-diagonal) matrix. The first term on the r.h.s. of (9) generates SPM. The second summation term with a $3 / 2$ multiplier generates XPM. The factor $3 / 2$ accounts for the polarizationaveraged value of XPM, which runs between a value $1\left(\vec{A}_{k}\right.$ orthogonal to $\vec{A}_{n}, k \neq n$ ) and the classic value 2 of scalar propagation [30]. SPM and XPM are both scalar effects, since they just depend on signal intensities through the diagonal matrix $\sigma_{0}$. The third matrix summation term in (9) generates all the polarization-dependence of the cross-channel interactions and is thus called XPolM [47]. XPolM can be seen as the natural generalization of the scalar phase rotation induced by XPM in the complex plane to a rotation over the Poincar sphere.

At each time instant, the exact solution of (9) when $\mathrm{SPM}+\mathrm{XPM}+\mathrm{XPolM}$ are simultaneously active is (we omit the time dependence, since the nonlinear step is memoryless):

$$
\vec{A}_{n}(z)=e^{\frac{-j \bar{\gamma} z\left(A_{n}^{2}(0)+3 \sum_{k \neq n} A_{k}^{2}(0)\right)}{2}} \underline{e}^{-\frac{j \bar{\gamma} z}{2}\left(\vec{s}_{t}(0) \cdot \vec{\sigma}\right)} \vec{A}_{n}(0)
$$

where $\vec{s}_{t}(z)=\vec{s}_{t}(0) \triangleq \sum_{k} \vec{a}_{k}(0)$ is the real pivot vector (a constant in $z$ ), and $\underline{e}^{(.)}$denotes a matrix exponential, which can be computed for the generic real vector $\vec{v}$ of norm $|\vec{v}|$ as [46]:

$$
\underline{e}^{-j(\vec{v} \cdot \vec{\sigma})}=\cos (|\vec{v}|) \sigma_{0}-j \sin (|\vec{v}|)\left(\frac{\vec{v}}{|\vec{v}|} \cdot \vec{\sigma}\right) .
$$

Using the proposed selective activation of nonlinearities, the exact solution of the Manakov nonlinear step (9) for the cases labeled SPM, XPM, XPolM and XCI in the results section is

$$
\begin{aligned}
\vec{A}_{n, \mathrm{SPM}}(z) \triangleq e^{-j \bar{\gamma} A_{n}^{2}(0) z} \vec{A}_{n}(0) \\
\vec{A}_{n, \mathrm{XPM}}(z) \triangleq e^{-j \bar{\gamma} \frac{3}{2} \sum_{k \neq n} A_{k}^{2}(0) z} \vec{A}_{n}(0) \\
\vec{A}_{n, \mathrm{XPolM}}(z) \triangleq e^{\frac{j \bar{\gamma} A_{n}^{2}(0) z}{2}} \underline{e}^{-\frac{j \bar{\gamma}}{2}\left(\vec{s}_{t}(0) \cdot \vec{\sigma}\right) z} \vec{A}_{n}(0) \\
\vec{A}_{n, \mathrm{XCI}}(z) \triangleq e^{-j \bar{\gamma} \frac{3}{2} \sum_{k \neq n} A_{k}^{2}(0) z} \vec{A}_{n, \mathrm{XPolM}}(z) .
\end{aligned}
$$

Note that eq. (9) is indeed a set of $N_{\text {ch }}$ coupled differential equations, which we refer to as SFP [27]. Hence the split-step Fourier simulations with SFP that we used to generate the NLT curves labeled SPM, XPM, XPolM and XCI consist of forming a matrix whose columns are the fields $\vec{A}_{k}$ discretized over the fast Fourier transform grid corresponding to a frequency window equal to the channel spacing ${ }^{7} \Delta f$. Then the matrix is propagated along $z$ such that at each step:

i) independent Gaussian ASE noise samples are added at each grid point if the $z$ coordinate corresponds to the position of an EDFA;

ii) the $N_{\text {ch }}$ signal fields are impaired by linear effects only (attenuation, chromatic dispersion);

iii) the $N_{\text {ch }}$ fields are impaired by nonlinear effects only, according to (11)-(14).

Note that point i) is applied only in the case of distributed noise, and amounts to adding to each channel a white ASE over a bandwidth equal to the channel spacing $\Delta f$.

At small bandwidth efficiency $\eta \ll 1$ the SFP is computationally faster than the UFP, where the WDM signal (8) is treated as a unique channel and the Manakov step is just $\frac{\partial \vec{A}}{\partial z}=-i \bar{\gamma} A^{2} \vec{A}$ but requires a much shorter sampling time, typically of the order of the inverse of three times the WDM occupied bandwidth, to account for at least first-order FWM. The curves labeled WDM in all numerical results were obtained with the UFP and therefore do account for linear channel crosstalk as well as all nonlinear effects, including FWM and nonlinear spectral broadening, which are instead not captured by the SFP solutions. Hence the $\mathrm{SPM}+\mathrm{XPM}+\mathrm{XPolM}$ simulations using SFP are less accurate than the WDM simulations using UFP at bandwidth efficiencies $\eta$ approaching the Nyquist-WDM value 1.

\section{ACKNOWLEDGMENT}

The authors would like to thank S. Jansen for asking questions that triggered the search of the optimal symbol rate from NLT curves, and G. Charlet for his insightful comments.

\section{REFERENCES}

[1] L. B. Du and A. J. Lowery, "Optimizing the subcarrier granularity of coherent optical communications systems," Opt. Exp., vol. 19, no. 9, pp. 8079-8084, Apr. 2011.

[2] A. D. Ellis et al., "Future directions to realise ultra- high bit rate transmission systems," presented at the 15th Optoelectron. Commun. Conf., Sapporo, Japan, 2010, Paper 8B3-1.

\footnotetext{
${ }^{7}$ In our implementation, a window $>3 \Delta f$ is allocated to avoid aliasing due to nonlinear broadening.
} 
[3] S. L. Jansen, "Multi-carrier approaches for next-generation transmission: Why, where and how?" presented at the Opt. Fiber Commun. Conf. Expo./ Nat. Fiber Optic Eng. Conf., Los Angeles, CA, USA, 2012, Paper OTh1B.1.

[4] Y. Tang, W. Shieh, and B. S. Krongold, "DFT-spread ofdm for fiber nonlinearity mitigation," IEEE Photon. Technol. Lett., vol. 22, no. 16, pp. 1250-1252, Aug. 2010.

[5] F. Zhang, C. Yang, X. Fang, T. Zhang, and Z. Chen, "Nonlinear performance of multigranularity orthogonal transmission systems with frequency division multiplexing," Opt. Exp., vol. 21, no. 5, pp. 6115-6130, Mar. 2013.

[6] P. Poggiolini et al., "Performance dependence on channel baud-rate of PM-QPSK systems over uncompensated links," IEEE Photon. Technol. Lett., vol. 23, no. 1, pp. 15-17, Jan. 2011.

[7] E. Palkopoulou et al., "Nyquist-WDM-based flexible optical networks: Exploring physical layer design parameters," J. Lightw. Technol., vol. 31, no. 14, pp. 2332-2339, Jul. 2013.

[8] C. Behrens, R. I. Killey, S. J. Savory, M. Chen, and P. Bayvel, "Nonlinear transmission performance of higher-order modulation formats," IEEE Photon. Technol. Lett., vol. 23, no. 6, pp. 377-379, Mar. 2011.

[9] P. Poggiolini, Y. Jiang, A. Carena, G. Bosco, and F. Forghieri, "Analytical results on system maximum reach increase through symbol rate optimization," presented at the Opt. Fiber Conf., Los Angeles, CA, USA, 2015, Paper Th3D.6.

[10] A. Bononi, N. Rossi, and P. Serena, "Transmission limitations due to fiber nonlinearity," presented at the Opt. Fiber Commun. Conf. Expo./ Nat. Fiber Optic Eng. Conf., Los Angeles, CA, USA, 2011, Paper OWO7.

[11] E. Grellier, J.-C. Antona, and S. Bigo, "Global criteria to account for tolerance to nonlinearities of highly dispersive systems," IEEE Photon. Technol. Lett., vol. 22, no. 10, pp. 685-687, May 2010.

[12] A. J. Stark et al., "Scaling $112 \mathrm{~Gb} / \mathrm{s}$ optical networks with the nonlinear threshold metric," J. Lightw. Technol., vol. 30, no. 9, pp. 1291-1298, May 2012.

[13] A. Bononi, N. Rossi, and P. Serena, "On the nonlinear threshold versus distance in long-haul highly-dispersive coherent systems," Opt. Exp., vol. 20, no. 26, pp. B204-B216, Dec. 2012.

[14] J.-C. Antona and S. Bigo, "Physical design and performance estimation of heterogeneous optical transmission systems," Comptes Rendus Physique, vol. 9/10, pp. 963-984, Nov./Dec. 2008.

[15] A. Bononi, N. Rossi, and P. Serena, "Performance dependence on channelbaudrate of coherent single-carrier WDM systems," presented at the 39th Eur. Conf. Exhib. Opt. Commun., London, U.K., 2013, Paper Th.1.D.5.

[16] C. Xie, "Interchannel nonlinearities in coherent polarization-divisionmultiplexed quadrature-phase-shift-keying systems," IEEE Photon. Technol. Lett., vol. 21, no. 5, pp. 274-276, Mar. 2009

[17] O. Bertran-Pardo et al., "PDM-QPSK: On the system benefits arising from temporally interleaving polarization tributaries at $100 \mathrm{~Gb} / \mathrm{s}$," Opt. Exp., vol. 17, no. 22, pp. 19902-19907, Oct. 2009.

[18] A. Bononi, P. Serena, N. Rossi, and D. Sperti, "Which is the dominant nonlinearity in long-haul PDM-QPSK coherent transmissions?," presented at the 36th Eur. Conf. Exhib. Opt. Commun., Torino, Italy, 2010, Paper Th.10.E.1.

[19] P. Poggiolini, "The GN model of nonlinear propagation in uncompensated coherent optical systems," J. Lightw. Technol., vol. 30, no. 24, pp. 3857-3879, Dec. 2012

[20] P. Serena and A. Bononi, "An alternative approach to the Gaussian noise model and its system implications," J. Lightw. Technol., vol. 31, no. 22, pp. 3489-3499, Nov. 2013.

[21] A. Carena, G. Bosco, V. Curri, P. Poggiolini, and F. Forghieri, "Impact of the transmitted signal initial dispersion transient on the accuracy of the GN-model of nonlinear propagation," presented at the 39th Eur. Conf. Exhib. Opt. Commun., London, U.K., 2013, Paper Th.1.D.4

[22] R. Dar, M. Feder, A. Mecozzi, and M. Shtaif, "Properties of nonlinear noise in long, dispersion-uncompensated fiber links," Opt. Exp., vol. 21, no. 22, pp. 25685-25699, Jul. 2013.

[23] A. Carena et al., "EGN model of nonlinear fiber propagation," Opt. Exp., vol. 22, no. 13, pp. 16335-16362, Jun. 2014.

[24] S. Kilmurray, T. Fehenberger, P. Bayvel, and R. I. Killey, "Comparison of the nonlinear transmission performance of quasi-Nyquist WDM and reduced guard interval OFDM," Opt. Exp., vol. 20, no. 4, pp. 4198-4205, Feb. 2012.

[25] S. Bigo, "PDM-QPSK transmission experiments over transoceanic distance," presented at the IEEE Photonics Soc. Summer Top. Meet. Series, Playa del Carmen, Mexico, Jul. 2010, Paper TuA2.1.
[26] F. Vacondio et al., "On nonlinear distortions of highly dispersive optical coherent systems," Opt. Exp., vol. 20, no. 2, pp. 1022-1032, Jan. 2012.

[27] P. Serena, M. Bertolini, and A. Vannucci, (2009), "Optilux toolbox," [Online]. Available: http://www.optilux.sourceforge.net

[28] A. Bononi, P. Serena, and A. Orlandini, "A unified design framework for single-channel dispersion-managed terrestrial systems," J. Lightw. Technol., vol. 26, no. 22, pp. 3617-3631, Nov. 2008.

[29] C. R. Menyuk and B. S. Marks, "Interaction of polarization mode dispersion and nonlinearity in optical fiber transmission systems," J. Lightw. Technol., vol. 24, no. 7, pp. 2806-2826, Jul. 2006.

[30] M. Winter, C. A. Bunge, D. Setti, and K. Petermann, "A statistical treatment of cross-polarization modulation in DWDM systems," J. Lightw. Technol., vol. 27, no. 17, pp. 3739-3751, Sep. 2009.

[31] T. Pfau and S. Hoffmann, "Hardware-efficient coherent digital receiver concept with feedforward carrier recovery for -QAM constellations," $J$. Lightw. Technol., vol. 27, no. 8, pp. 989-999, Apr. 2009.

[32] A. Bononi, P. Serena, and N. Rossi, "Nonlinear signal-noise interactions in dispersion-managed links with various modulation formats," Opt. Fiber Technol., vol. 16, no. 2, pp. 73-85, Mar. 2010.

[33] A. Carena, V. Curri, G. Bosco, P. Poggiolini, and F. Forghieri, "Modeling of the impact of non-linear propagation effects in uncompensated optical coherent transmission links," J. Lightw. Technol., vol. 30, no. 10, pp. 1524-1539, May 2012.

[34] A. Bononi, M. Bertolini, P. Serena, and G. Bellotti, "Cross-phase modulation induced by OOK channels on higher-rate DQPSK and coherent QPSK channels," J. Lightw. Technol., vol. 27, no. 18, pp. 3974-3983, Sep. 2009.

[35] E. Ip and J. M. Kahn, "Feedforward carrier recovery for coherent optical communications," J. Lightw. Technol., vol. 25, no. 9, pp. 2675-2692, Sep. 2007.

[36] G. Bosco et al., "Performance prediction for WDM PM-QPSK transmission over uncompensated links," presented at the Opt. Fiber Commun. Conf. Expo./ Nat. Fiber Optic Eng. Conf., Los Angeles, CA, USA, 2011, Paper OThO7.

[37] E. Grellier and A. Bononi, "Quality parameter for coherent transmissions with Gaussian-distributed nonlinear noise," Opt. Exp., vol. 19, no. 13, pp. 12781-12788, Jun. 2011.

[38] C. Xia and D. Van Den Borne, "Impact of the channel count on the nonlinear tolerance in coherently-detected POLMUX-QPSK modulation," presented at the Opt. Fiber Commun. Conf. Expo./ Nat. Fiber Optic Eng. Conf., Los Angeles, CA, USA, 2011, Paper OWO1.

[39] J. Renaudier et al., "On the required number of WDM channels when assessing performance of $100 \mathrm{~Gb} / \mathrm{s}$ coherent PDM-QPSK overlaying legacy systems," presented at the 35th Eur. Conf. Opt. Commun., Vienna, Austria, Sep. 2009, Paper Tu3.4.5.

[40] Y.-H. Wang and I. Lyubomirsky, "Impact of DP-QPSK pulse shape in nonlinear $100 \mathrm{G}$ transmission," J. Lightw. Technol., vol. 28, no. 18, pp. 2750-2756, Sep. 2010.

[41] A. Bononi, O. Beucher, and P. Serena, "Single- and cross-channel nonlinear interference in the Gaussian noise model with rectangular spectra," Opt. Exp., vol. 21, no. 26, pp. 32254-32268, Dec. 2013.

[42] A. Bononi, O. Beucher, and P. Serena, "Erratum: Single- and cross-channel nonlinear interference in the Gaussian noise model with rectangular spectra," viXra:1504.0188v1, [Digital Signal Processing] (2015).

[43] P. Poggiolini et al. "The GN model of fiber non-linear propagation and its applications," J. Lightw. Technol., vol. 32, no. 4, pp. 694-721, Feb. 2014

[44] P. Serena and A. Bononi, "A time-domain extended Gaussian noise model,” J. Lightw. Technol., vol. 33, no. 7, pp. 1459-1472, Apr. 2015.

[45] P. Poggiolini, A. Carena, V. Curri, G. Bosco, and F. Forghieri, "Analytical modeling of nonlinear propagation in uncompensated optical transmission links," IEEE Photon. Technol. Lett., vol. 23, no. 11, pp. 742-744, Jun. 2011.

[46] J. P. Gordon and H. Kogelnik, "PMD fundamentals: Polarization mode dispersion in optical fibers," Proc. Nat. Acad. Sci., vol. 97, pp. 4541-4550, Apr. 2000.

[47] M. Karlsson and H. Sunnerud, "Effects of nonlinearities on PMD-induced system impairments," J. Lightw. Technol., vol. 24, no. 11, pp. 4127-4137, Nov. 2006.

Authors' biographies not available at the time of publication. 